\title{
Lesión masiva del manguito rotador: tratamiento artroscópico con reconstrucción capsular superior
}

\author{
Massive rotator cuff injury: arthroscopic treatment with upper capsular reconstruction \\ Torres-Loaiza JW,* Velasco A,* Villegas R, ${ }^{\ddagger}$ Salazar G,* Escobar G* \\ Hospital «Pablo Arturo Suárez». Quito-Ecuador.
}

RESUMEN. Las lesiones masivas del manguito rotador (LMMR) son aquellas roturas de más de $5 \mathrm{~cm}$ ya sea el plano anteroposterior o lateromedial. Algunos autores las consideran como roturas completas de al menos dos tendones del maguito rotador. Se presenta el caso clínico de un varón de 63 años con este tipo de lesión que se resolvió mediante la técnica de reconstrucción de cápsula superior, se muestran detalles técnicos y un poco de la revisión de la literatura para llevar a cabo el procedimiento.

Palabras clave: Manguito rotador, masiva, tratamiento, cápsulas superior.

\section{Introducción}

Las lesiones masivas del manquito rotador (LMMR) son aquellas roturas de más de $5 \mathrm{~cm}$ ya sea el plano anteroposterior o lateromedial. ${ }^{1}$ Gerber las define como roturas completas de al menos dos tendones del maguito rotador. ${ }^{2}$ A pesar de que no existe un consenso sobre cuál clasificación/definición es la mejor, es importante la interpretación del patrón de rotura para definir la situación clínica del paciente. ${ }^{1}$ Son comunes en la población adulta, teniendo una prevalencia de $22 \%$ en personas mayores de 65 años y se encuentran hasta en $40 \%$ de todas las roturas de manguito rotador. Además, estas lesiones poseen un alto potencial de ser irreparables. ${ }^{3,4}$
ABSTRACT. Massive rotator cuff injuries (LMMRs) are those breaks of more than $5 \mathrm{~cm}$ either the anteroposterior plane, or lateromedial plane. Some authors consider them to be complete ruptures of at least two rotator cuff tendons. The clinical case of a 63-year-old man with this type of injury that was resolved by the superior capsule reconstruction technique is presented, technical details and some of the literature review to carry out the procedure are shown.

Keywords: Rotator cuff, massive, treatment, superior capsule.

Clínicamente se acompañan de dolor, otros síntomas observados son debilidad, rigidez, crepitación o inestabilidad. ${ }^{5,6,7}$ La cronicidad del dolor puede estar relacionada con la capacidad de recuperación, debido a que suelen estar asociadas con cambios degenerativos. ${ }^{5,8}$ Las comorbilidades asociadas son diabetes mellitus, índice de masa corporal alto, uso crónico de corticosteroides orales y otras tendinopatías. ${ }^{7,9,10,11,12,13,14}$

Los estudios de imágenes son determinantes; ${ }^{4,15}$ se deben identificar signos de artrosis glenohumeral, el ascenso de la cabeza humeral puede ser determinado a través del intervalo acromiohumeral, que corresponde a la distancia entre el extremo superior de la cabeza humeral y la superficie inferior del acromion en una radiografía anteroposterior verda-

\footnotetext{
Dirección para correspondencia:

Dr. Jorge W Torres Loaiza

José Muñecas 180 y José Paredes. EC170104

5930995221739 (Ecuador)

E-mail: drjorgewtorres@gmail.com
}

* Médico Especialista Traumatología Artroscopía. Hospital General «Pablo Arturo Suárez», Quito-Ecuador.

‡ Médico residente tercer de postgrado en Ortopedia y Traumatología. 
dera de hombro con rotación neutra del húmero. ${ }^{15,16,17,18} \mathrm{La}$ especificidad y la sensibilidad de la ecografía es operador dependiente. ${ }^{19}$ La resonancia magnética simple tiene una sensibilidad de 94\% y especificidad de 93\% para la detección de roturas completas del maguito rotador, ${ }^{20,21,22}$ además permite una valoración detallada del grado de infiltración grasa, morfología de la lesión y retracción tendinosa. ${ }^{15}$

\section{Caso clínico}

Paciente masculino de 63 años, diestro. Sin antecedentes personales clínicos ni quirúrgicos. Presenta un cuadro de dolor de hombro izquierdo de dos años de evolución, posterior a un trauma directo asociado a una caída. Inicialmente

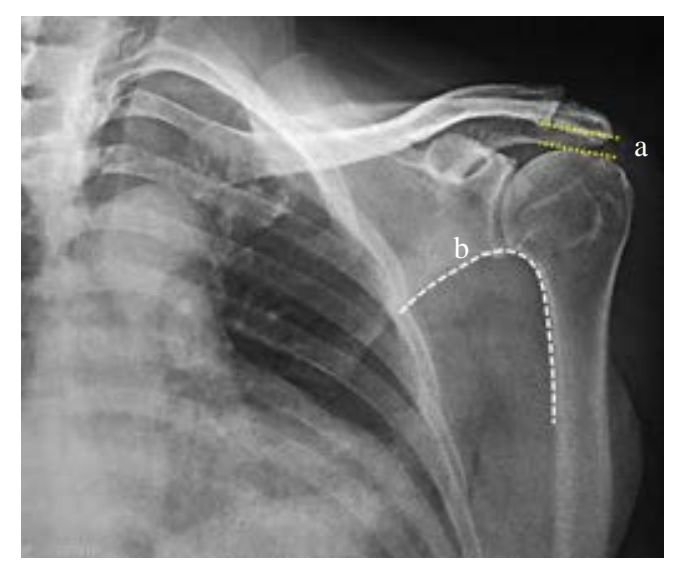

Figura 1: Radiografía anteroposterior de hombro izquierdo. a) Intervalo acromiohumeral: $8 \mathrm{~mm}$. b) Continuidad del arco escapulohumeral. con dolor de moderada intensidad (escala análoga de dolor (EVA): 4/10), lancinante, con esporádica irradiación a cara externa y superior de brazo, éste se intensificaba en las noches o con las actividades que requerían la elevación del codo por encima del hombro. Inicialmente tratado con analgésicos y fisioterapia, con lo cual presentaba remisión temporal. Al cabo de 18 meses después presenta exacerbación (EVA: 6/10) y limitación para la realización de actividades diarias que requieren la flexión y abducción del brazo. Valor de SST: 5.

Al examen físico: peso: $74 \mathrm{~kg}$, talla: $1.54 \mathrm{~m}$, índice de masa corporal: 31.2. Hombro izquierdo:

Movilidad activa: flexión: $40^{\circ}$, extensión: $20^{\circ}$, rotación interna L4, rotación externa: $30^{\circ}$, abducción: $80^{\circ}$, aducción: $40^{\circ}$. Movilidad pasiva: flexión: $60^{\circ}$, extensión: $20^{\circ}$, rotación interna L2, rotación externa: $30^{\circ}$, abducción: $100^{\circ}$, aducción: 40. Pruebas específicas: Hawkings Kennedy: Positivo, Neer: positivo, Speed: positivo, Jobe: positivo, Belly Press: positivo, lateral rotation lag: positivo, internal rotation lag: positivo.

La radiografía anteroposterior de hombro muestra ausencia de signos radiográficos de artrosis glenohumeral y cambios degenerativos iniciales en la articulación acromioclavicular (Figura 1). La resonancia magnética mostró una lesión completa del maguito rotador con compromiso de los tendones del supraespinoso, infraespinoso y subescapular, con una retracción a nivel de la articulación acromioclavicular. Ausencia del tendón largo del bíceps en la corredera bicipital. Signos de infiltración grasa del supraespinoso (Figura 2).

Se realizó una reconstrucción capsular superior con aloinjerto de tendón de Aquiles de $60 \times 3.5$ mm. El proce-
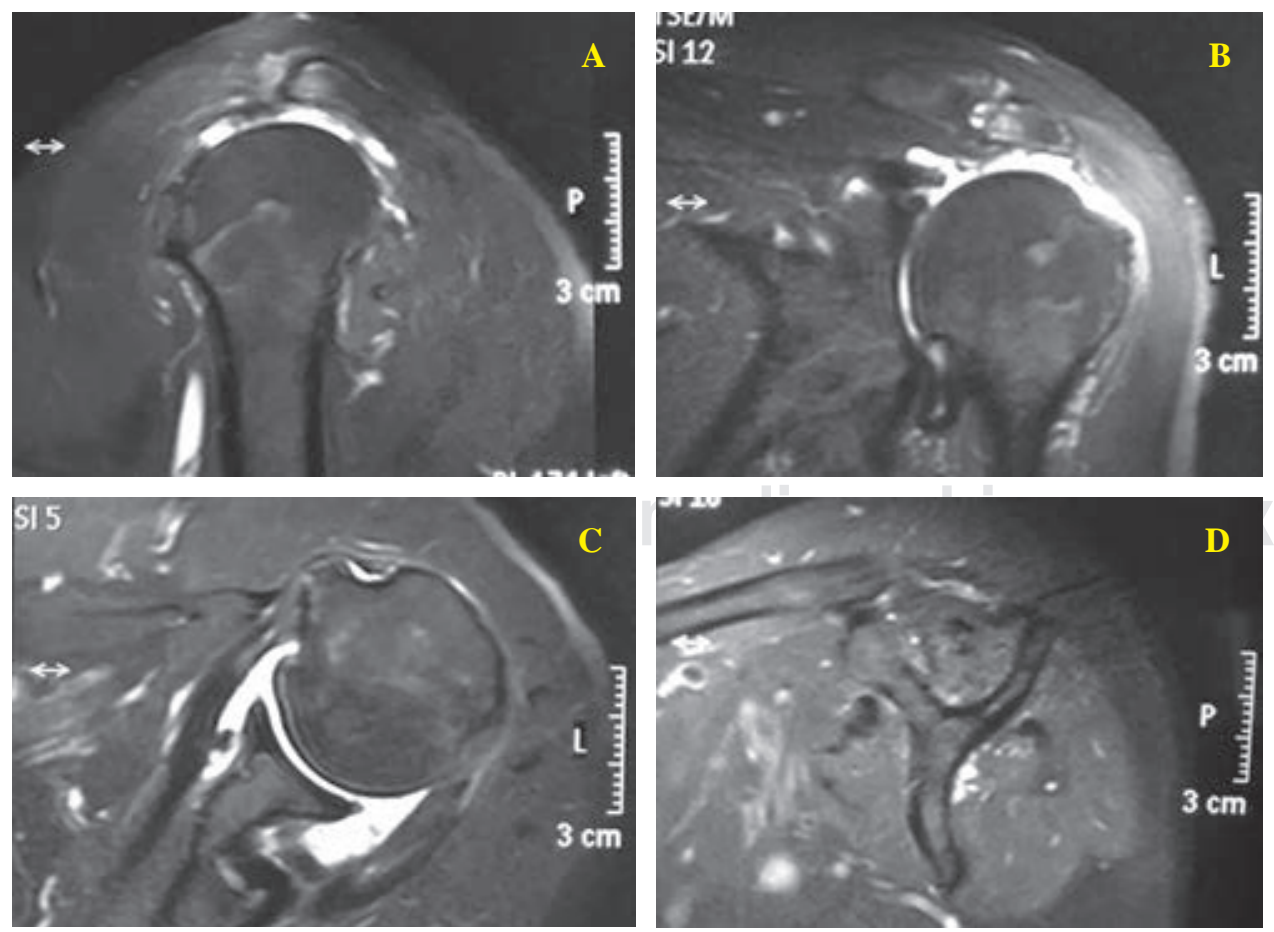

B

Figura 2:

Resonancia magnética simple de hombro izquierdo. A) Corte sagital. Rotura completa de $38 \mathrm{~mm} \mathrm{B)}$ Corte coronal: rotura completa con retracción de $59 \mathrm{~mm}$. C) Corte axial. Nótese la ausencia del tendón del bíceps en la corredera bicipital. D) Corte sagital a nivel de cuerpo de escápula, se evidencia hipotrofia del músculo supraespinoso. 
dimiento fue llevado a cabo vía artroscópica utilizando dos anclas colocadas en el cuello de la glenoides y dos anclas colocadas en el húmero. En el control radiográfico postquirúrgico se visualizan las anclas utilizadas (Figura 3).

El paciente usó un inmovilizador de hombro por un período de 15 días, iniciando movimientos pendulares y movilidad pasiva asistida no forzados. Se realizó un control de resonancia magnética a las tres semanas de postoperado, en el cual se evidencia la presencia del aloinjerto en posición deseada. El paciente mostró mejoría de los arcos de movilidad (Figura 4), disminución considerable del dolor y un valor de SST: 8 en su control a las cuatro semanas.

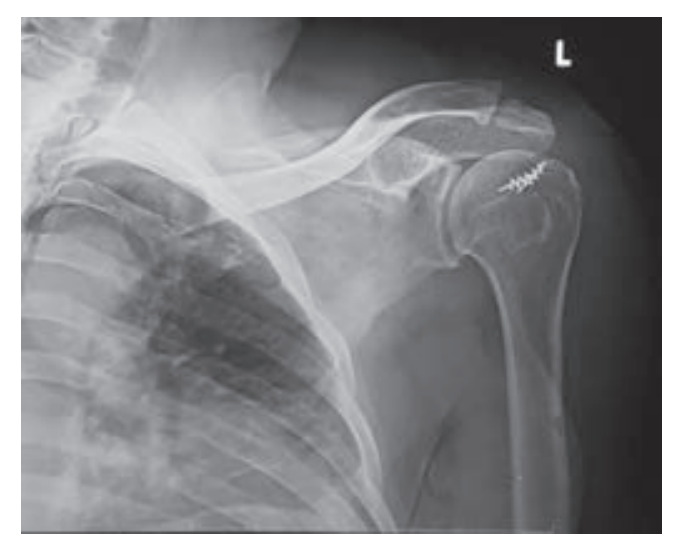

Figura 3: Control radiográfico postquirúrgico.

\section{Técnica quirúrgica}

Paciente en posición de silla de playa. Se inicia con un portal posterior, con el artroscopio de $30^{\circ}$ y un portal de trabajo anterolateral (Figura 5).

Una vez identificada la lesión se realiza la preparación del cuello de la glenoides en su superficie superior donde se colocaron dos anclas (Figura 6). La colocación de la guía debe estar a 2-3 mm medial al borde externo de la glenoides, con una inclinación de afuera hacia adentro para evitar la superficie articular.

En el húmero el ancla anterior debe ser colocada justo posterior a la corredera bicipital y el ancla posterior en el borde posterolateral del defecto del manguito rotatorio (Figura 7). A este punto se tendrán las cuatro anclas colocadas. Con ayuda del palpador se procede a tomar las medidas para la preparación final del injerto. Usando como referencia la colocación de las anclas se agregan $5 \mathrm{~mm}$ más al tamaño obtenido hacia anterior, posterior y medial; y, $10 \mathrm{~mm}$ hacia lateral. ${ }^{1}$ En el presente caso clínico fue de $46 \times 25 \mathrm{~mm}$.

Preparación del injerto: se solicitó un aloinjerto de tendón de Aquiles sin pastilla ósea. Se colocó una sutura en cada una de las esquinas para fijación. Las suturas de las anclas colocadas se recuperaron por el portal lateral y se recomienda el uso de una cánula flexible. Se debe realizar el paso de una sutura por cada ancla colocada en la esquina que va a ocupar el injerto dentro del espacio subacromial.
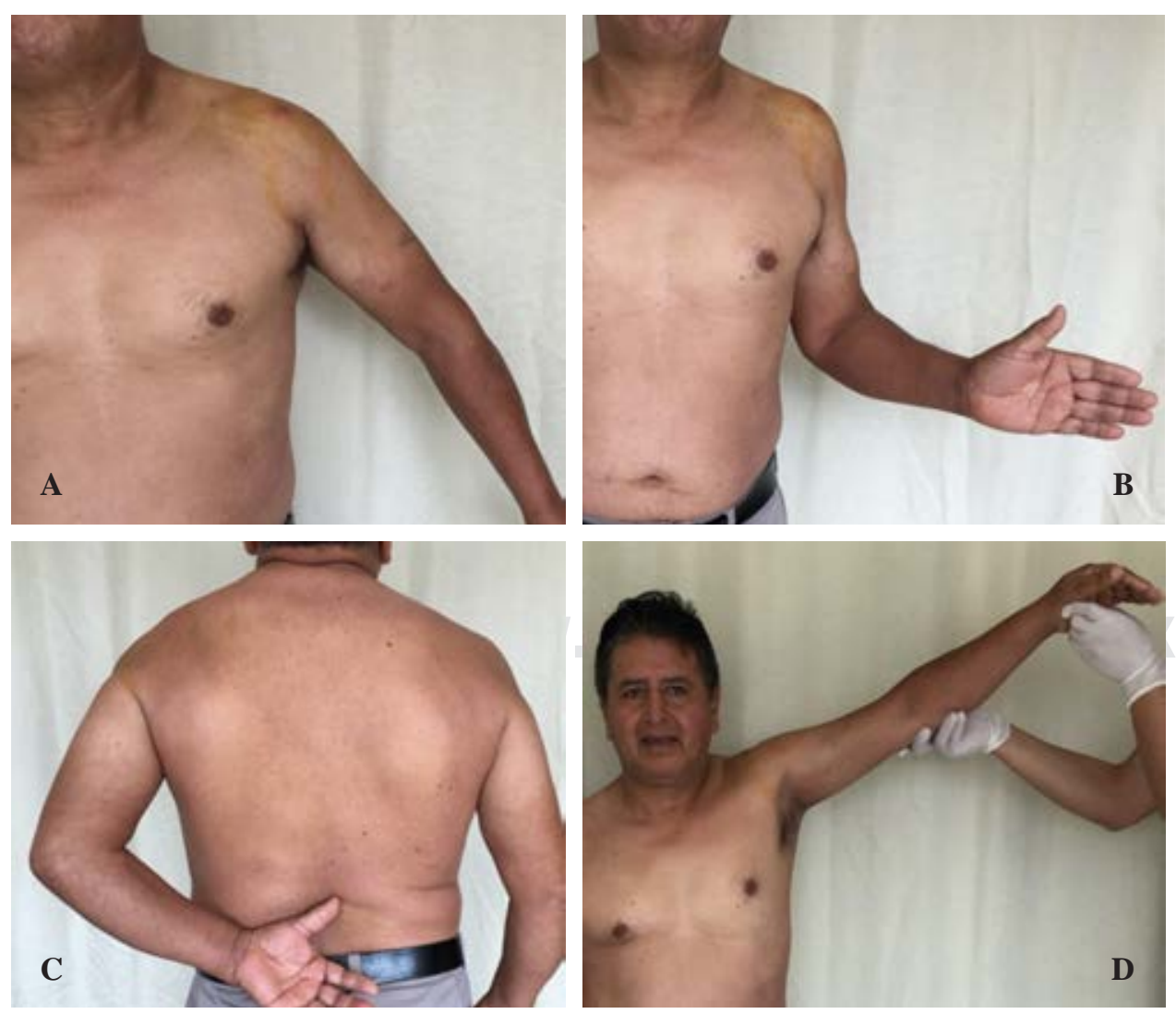

Figura 4:

Arcos de movilidad activa y pasiva de hombro. A) Abducción activa. B) Rotación externa activa. C) Rotación interna activa. D) Abducción pasiva. 


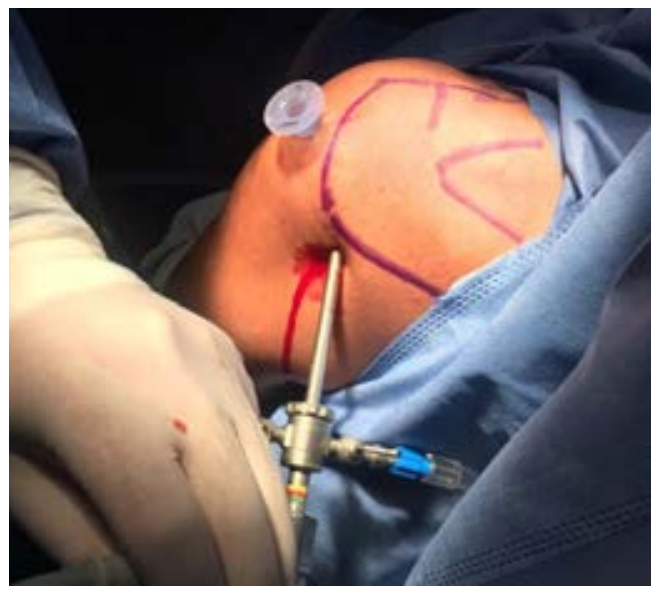

Figura 5: Ubicación de portales.
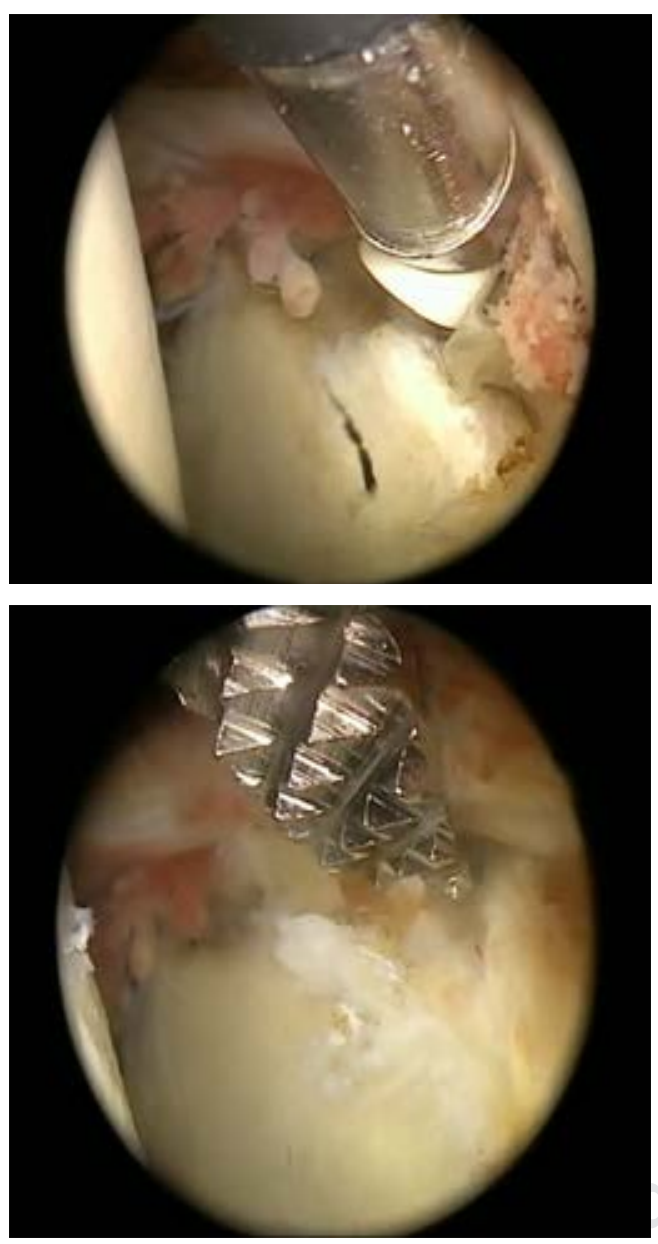

Figura 6: Preparación de glenoides.

En primera instancia se colocan las suturas correspondientes a las anclas de glenoides.

Una vez que ingrese el injerto en la cavidad subacromial, la visualización será difícil, debiendo asegurar el paso de las suturas de las anclas colocadas en el húmero a su correspondiente esquina en el aloinjerto. Para esto se puede realizar el paso de la sutura previo a la introducción del injerto y evitar el cruce de éstas, de tal modo que ingresara primero el extremo que se encuentra con las suturas colocadas en la glenoides y se hace un nudo en cada una de ellas y posteriormente se hará un nudo en las anclas anterior y posterior del húmero. Para complementar se puede realizar una sutura entre las dos anclas de la glena y las dos anclas del húmero (Figura 8).

\section{Discusión}

La reconstrucción capsular superior (RCS) es una técnica destinada para las LMMR irreparables, consiste en un procedimiento artroscópico mediante el cual se coloca un injerto que servirá de interposición entre la cabeza humeral y el acromion, fijado al cuello de la glenoides y al troquiter por medio de dispositivos ortopédicos. ${ }^{23}$ Deben considerarse otros gestos quirúrgicos como reparo del subescapular, coracoplastía y tenodesis o tenotomía del bíceps, mismos que precederán a la realización de la RCS. ${ }^{23}$ En el caso presentado existió una rotura espontánea del tendón largo del bíceps previo a la intervención quirúrgica.

Estudios biomecánicos han demostrado que la RCS restaura la estabilidad capsular superior y restringe la traslación superior del húmero, obteniendo resultados similares a la colocación del espaciador subacromial y superiores a la aumentación del supraespinoso. ${ }^{24,25}$

La información disponible es limitada debido a que los reportes son escasos sin resultados a largo plazo..$^{26,27}$ No obstante, los resultados clínicos a un período de uno y dos años han sido prometedores. ${ }^{28,29,30,31,32,33}$ Por tal motivo, se dificulta identificar con exactitud a los pacientes que se beneficiarían de esta técnica; sin embargo, se considera como una opción terapéutica para pacientes con dolor no tolerable en quienes el tratamiento conservador es fallido y presentan un LMMR irreparable, con una artropatía glenohumeral mínima o ausente (grado 1-2 de Hamada), con un tendón del subescapular competente/reparable y funcionalidad adecuada del deltoides. ${ }^{31}$ Se han descrito varios tipos de injertos/

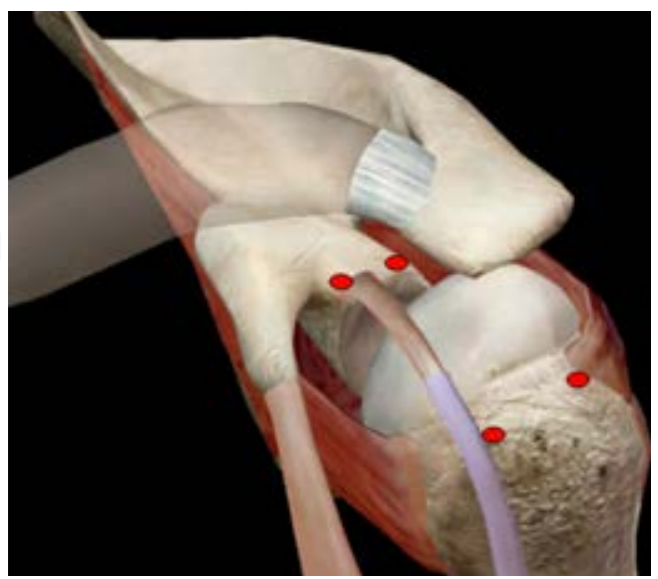

Figura 7: Ubicación final de los anclajes. 

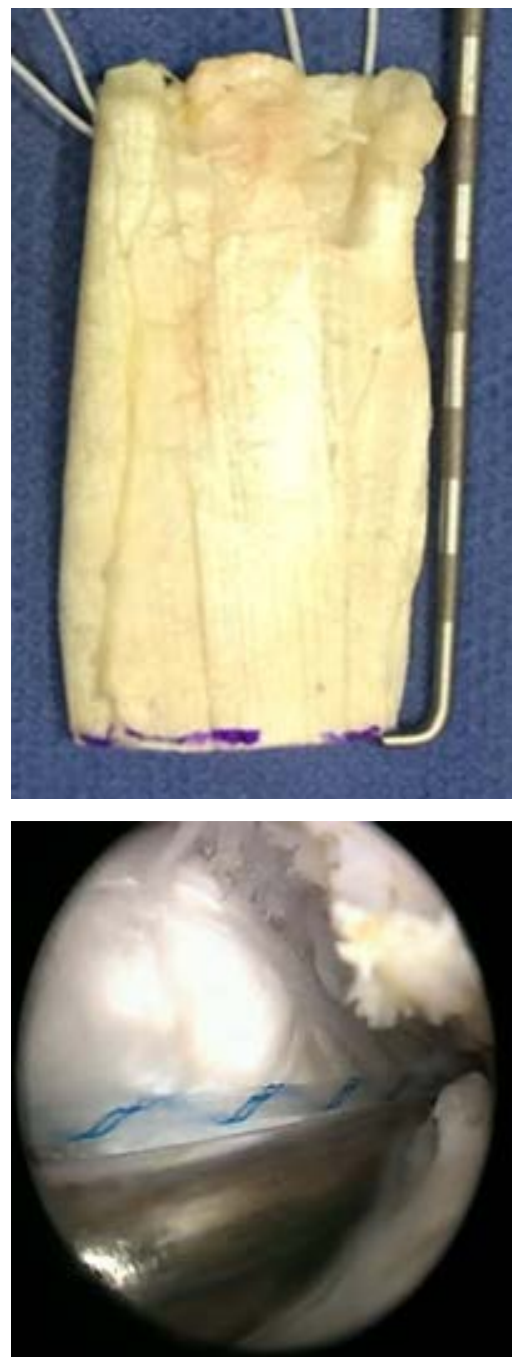

Figura 8: Preparación y vista artroscópica del injerto.

dispositivos para RCS, entre los que se mencionan: tensor de la fascia lata, ${ }^{28}$ dérmicos, ${ }^{30}$ isquiotibiales, ${ }^{34}$ entre otros. ${ }^{35}$ En el caso clínico presentado se utilizó aloinjerto de tendón de Aquiles debido al grosor que éste posee. Los resultados obtenidos a corto plazo fueron beneficiosos en términos de diminución del dolor, mejoría de la funcionalidad y calidad de vida como se ha demostrado en otros estudios.

\section{Bibliografía}

1. Burkhart SS, Denard PJ, Adams CR, Brady PC, Hartzler RU. Arthroscopic superior capsular reconstruction for massive irreparable rotator cuff repair. Arthrosc Tech. 2016; 5(6): e1407-18.

2. Gerber C, Fuchs B, Hodler J. The results of repair of massive tears of the rotator cuff. J Bone Joint Surg Am. 2000; 82(4): 505-15.

3. Fehringer EV, Sun J, VanOeveren LS, Keller BK, Matsen FA. Fullthickness rotator cuff tear prevalence and correlation with function and co-morbidities in patients sixty-five years and older. J Shoulder Elb Surg. 2008; 17(6): 881-5.

4. Bedi A, Dines J, Warren RF, Dines DM. Massive tears of the rotator cuff. J Bone Joint Surg Am. 2010; 92(9): 1894-908.

5. Hsu J, Gee A, Lippitt S, Matsen FA. The Rotator Cuff. In: Rockwood C, Matsen FA, Wirth M, Lippitt S, Fehringer E, Sperling J, editors.
Rockwood and Matsen's the shoulder. 5th ed. Philadelphia: Elsevier; 2018, 651-719.

6. Jeong J, Shin DC, Kim TH, Kim K. Prevalence of asymptomatic rotator cuff tear and their related factors in the Korean population. $J$ Shoulder Elb Surg. 2017; 26(1): 30-5.

7. Mathiasen R, Hogrefe C. Evaluation and management of rotator cuff tears: a primary care perspective. Curr Rev Musculoskelet Med. 2018; 11(1): 72-6.

8. Largacha Ponce de León M, Valbuena Bernal FJ, Alajmo Freire G. Lesiones masivas reparables del manguito de los rotadores. Revisión de conceptos actuales. Rev Colomb Ortop y Traumatol. 2016; 30: 56-62.

9. Titchener AG, White JJE, Hinchliffe SR, Tambe AA, Hubbard RB, Clark DI. Comorbidities in rotator cuff disease: a case-control study. $J$ Shoulder Elb Surg. 2014; 23(9): 1282-8.

10. Franceschi F, Papalia R, Paciotti M, Franceschetti E, Di Martino A, Maffulli N, et al. Obesity as a risk factor for tendinopathy: a systematic review. Int J Endocrinol. 2014; 2014: 1-10.

11. Membrilla-Mesa MD, Tejero-Fernández V, Cuesta-Vargas AI, ArroyoMorales M. Validation and reliability of a Spanish version of simple shoulder test (SST-Sp). Qual Life Res. 2015; 24(2): 411-6.

12. Collin P, Matsumura N, Lädermann A, Denard PJ, Walch G. Relationship between massive chronic rotator cuff tear pattern and loss of active shoulder range of motion. J Shoulder Elb Surg. 2014; 23(8): 1195-202.

13. Hermans J, Luime JJ, Meuffels DE, Reijman M, Simel DL, BiermaZeinstra SMA. Does this patient with shoulder pain have rotator cuff disease? The rational clinical examination systematic review. JAMA. 2013; 310(8): 837-47.

14. Jain NB, Wilcox RB, Katz JN, Higgins LD. Clinical examination of the rotator cuff. PMR. 2013; 5(1): 45-56.

15. Thès A, Hardy P, Bak K. Decision-making in massive rotator cuff tear. Knee Surg Sport Traumatol Arthrosc. 2015; 23(2): 449-59.

16. Balke M, Liem D, Greshake O, Hoeher J, Bouillon B, Banerjee M. Differences in acromial morphology of shoulders in patients with degenerative and traumatic supraspinatus tendon tears. Knee Surgery, Sport Traumatol Arthrosc. 2016; 24(7): 2200-5.

17. Moor BK, Wieser K, Slankamenac K, Gerber C, Bouaicha S. Relationship of individual scapular anatomy and degenerative rotator cuff tears. J Shoulder Elbow Surg. 2014; 23(4): 536-41.

18. Goutallier D, Le Guilloux P, Postel JM, Radier C, Bernageau J, Zilber $\mathrm{S}$. Acromio humeral distance less than six millimeter: its meaning in full-thickness rotator cuff tear. Orthop Traumatol Surg Res. 2011; 97(3): 246-51.

19. Tse AK, Lam PH, Walton JR, Hackett L, Murrell GA. Ultrasound determination of rotator cuff tear repairability. Vol. 8, Núm. 1, Shoulder \& Elbow. 2016, 14-21.

20. Lenza M, Buchbinder R, Takwoingi Y, Johnston R V, Hanchard NC, Faloppa F. Magnetic resonance imaging, magnetic resonance arthrography and ultrasonography for assessing rotator cuff tears in people with shoulder pain for whom surgery is being considered. Cochrane Database Syst Rev. 2013; (9): CD009020.

21. Sellers TR, Abdelfattah A, Frankle MA. Massive rotator cuff tear: when to consider reverse shoulder arthroplasty. Curr Rev Musculoskelet Med. 2018; 11(1): 131-40.

22. Carver TJ, Kraeutler MJ, Smith JR, Bravman JT, McCarty EC. Nonarthroplasty surgical treatment options for massive, irreparable rotator cuff tears. Orthop J Sport Med. 2018; 6(11): 2325967118805385.

23. Burkhart SS, Denard PJ, Adams CR, Brady PC, Hartzler RU. Arthroscopic superior capsular reconstruction for massive irreparable rotator cuff repair. Arthrosc Tech. 2016; 5(6): e1407-18.

24. Mihata T, McGarry MH, Pirolo JM, Kinoshita M, Lee TQ. Superior capsule reconstruction to restore superior stability in irreparable rotator cuff tears. Am J Sports Med. 2012; 40(10): 2248-55.

25. Singh S, Reeves J, Langohr GDG, Johnson JA, Athwal GS. The subacromial balloon spacer versus superior capsular reconstruction in the treatment of irreparable rotator cuff tears: a biomechanical assessment. Arthrosc J Arthrosc Relat Surg. 2019; 35(2): 382-9.

26. Ekhtiari S, Adili AF, Memon M, Leroux T, Henry P, Bedi A, et al. Sources, quality, and reported outcomes of superior capsular 
reconstruction: a systematic review. Curr Rev Musculoskelet Med. 2019; 12(2): 173-80.

27. Catapano M, de SA D, Ekhtiari S, Lin A, Bedi A, Lesniak BP. Arthroscopic superior capsular reconstruction for massive, irreparable rotator cuff tears: a systematic review of modern literature. Arthroscopy. 2019; 35(4): 1243-53.

28. Mihata T, Watanabe C, Fukunishi K, Ohue M, Tsujimura T, Kinoshita M. Arthroscopic superior capsular reconstruction restores shoulder stability and function in patients with irreparable rotator cuff tears: a prospective study (SS-15). Arthrosc J Arthrosc Relat Surg. 2011; 27(5): e36-7.

29. Pennington W, Bartz B, Pauli J. Arthroscopic superior capsular reconstruction for the treatment of massive irreparable rotator cuff tears in the active patient: 1-2 year results. Arthroscopy. 2018; 34(6): P1764-73.

30. Denard PJ, Brady PC, Adams CR, Tokish JM, Burkhart SS. Preliminary results of arthroscopic superior capsule reconstruction with dermal allograft. Arthroscopy. 2018; 34(1): 93-9.
31. Frank RM, Cvetanovich G, Savin D, Romeo AA. Superior capsular reconstruction. JBJS Rev. 2018; 6(7): e10.

32. Hartzler RU, Burkhart SS. Superior capsular reconstruction. Orthopedics. 2017; 40(5): 271-80.

33. Sochacki KR, McCulloch PC, Lintner DM, Harris JD. Superior capsular reconstruction for massive rotator cuff tear leads to significant improvement in range of motion and clinical outcomes: a systematic review. Arthroscopy. 2019; 35(4): 1269-77.

34. Rosales-Varo AP, Zafra M, García-Espona MA, Flores-Ruiz MA, Roda O. Reconstrucción de la cápsula superior en las roturas irreparables del manguito mediante injerto autógeno de isquiotibiales. Rev Esp Cir Ortop Traumatol. 2019; 63(1): 1-6.

35. Wall KC, Toth AP, Garrigues GE. How to use a graft in irreparable rotator cuff tears: a literature review update of interposition and superior capsule reconstruction techniques. Curr Rev Musculoskelet Med. 2018; 11(1): 122-30. 